\title{
Ginsenoside Re Inhibits Osteoclast Differentiation in Mouse Bone Marrow-Derived Macrophages and Zebrafish Scale Model
}

\author{
Chan-Mi Park ${ }^{1,3,9}$, Hye-Min Kim ${ }^{1,4,9}$, Dong Hyun Kim ${ }^{1,4}$, Ho-Jin Han ${ }^{1,4}$, Haneul Noh ${ }^{1}$, Jae-Hyuk Jang ${ }^{1,4}$, Soo- \\ Hyun Park ${ }^{2}$, Han-Jung Chae ${ }^{2}$, Soo-Wan Chae ${ }^{2}$, Eun Kyoung Ryu ${ }^{5}$, Sangku Lee ${ }^{1}$, Kangdong Liu ${ }^{1,7}$, Haidan \\ Liu $^{1,8}$, Jong-Seog Ahn ${ }^{1,4}$, Young Ock Kim ${ }^{6}$, Bo-Yeon $\mathrm{Kim}^{1,4, *}$, and Nak-Kyun Soung ${ }^{1,4, *}$
}

\begin{abstract}
Ginsenosides, which are the active materials of ginseng, have biological functions that include anti-osteoporotic effects. Aqueous ginseng extract inhibits osteoclast differentiation induced by receptor activator of NF-кB ligand (RANKL). Aqueous ginseng extract produces chromatography peaks characteristic of ginsenosides. Among these peaks, ginsenoside $\mathrm{Re}$ is a major component. However, the preventive effects of ginsenoside Re against osteoclast differentiation are not known. We studied the effect of ginsenoside $\mathrm{Re}$ on osteoclast differentiation, RANKLinduced tartrate-resistant acid phosphatase (TRAP) activity, and formation of multinucleated osteoclasts in vitro. Ginsenoside Re hampered osteoclast differentiation in a dose-dependent manner. In an in vivo zebrafish model, aqueous ginseng extract and ginsenoside Re had antiosteoclastogenesis effects. These findings suggest that both aqueous ginseng extract and ginsenoside Re prevent bone resorption by inhibiting osteoclast differentiation. Ginsenoside Re could be important for promoting bone health.
\end{abstract}

\footnotetext{
${ }^{1}$ World Class Institute (WCl), and Chemical biology Research Center, Korean Research Institute of Bioscience and Biotechnology (KRIBB), Cheongju 28116, Korea, ${ }^{2}$ Clinical Trial Center for Functional Foods (CTCF2), Chonbuk National University Hospital, Jeonju 54907, Korea, ${ }^{3}$ Department of Biochemistry, College of Nature science, Chungnam National University, Daejeon 34134, Korea, ${ }^{4}$ Biomolecular Science, University of Science and Technology, Daejeon 34113, Korea, ${ }^{5}$ Center of Magnetic Resonance Research, Korea Basic Science Institute, Cheongju 28119, Korea, ${ }^{6}$ Department of Medicinal Crop Research Institute, National Institute of Horticultural \& Herbal Science, Rural Development Administration, Eumseong 27709, Korea, ${ }^{7}$ Present address: Basic Medical College, Zhengzhou University, Zhengzhou, 450001, China, ${ }^{8}$ Present address: Department of Cardiothoracic Surgery, Clinical Center for Gene Diagnosis and Therapy, The Second Xiangya Hospital, Central South University, Changsha, 410011, China, ${ }^{9}$ These authors are contributed equally to this work.

*Correspondence: soungnak@kribb.re.kr (NKS); bykim@kirbb.re.kr (BYK)
}

Received 25 April, 2016; revised 4 November, 2016; accepted 7 November, 2016; published online 6 December, 2016

Keywords: ginsenoside Re, osteoclasts, RANKL, zebrafish

\section{INTRODUCTION}

Panax ginseng Meyer commonly known as Korean ginseng, which belongs to the Araliaceae family. Ginseng has bioactive effects on human health; it is widely used to treat immune dysfunction, liver dysfunction, renal deficiency, and blood vesselrelated diseases of the heart and brain. The human life span has dramatically increased in recent years, and aging is associated with several diseases. Osteoporosis is a widespread disease that is especially serious in countries with a large population of older adults. In 2004, the World Health Organization reported that $\sim 75$ million people were affected by osteoporosis (World Health Organization, 2004). Studies have examined the effects of ginseng-containing herbal extracts from ginseng and ginseng-related plants such as Siberian ginseng on pathologic bone remodeling (Kropotov et al., 2002; Lee et al., 2010; Siddiqi et al., 2013). Little is known, however, about the effects of ginseng on bone health (Polan et al., 2004).

In vertebrates, bone health is maintained by bone formation and resorption. Many bone disorders result from an imbalance between these two mechanisms (Goltzman, 2002). Osteoblasts originate from mesenchymal stem cells, whereas osteoclasts are formed from macrophages derived from hematopoietic stem cells. Osteoclasts are multinucleated cells formed by fusion of mononuclear macrophages (Boyle et al., 2003). Osteoblast cells affect osteoclast differentiation through macrophage colony-stimulating factor-1 (MCSF-1) and receptor activator of $\mathrm{NF}-\mathrm{kB}$ ligand (RANKL). Both are sufficient and necessary for osteoclast differentiation (Kong et al., 1999; Tanaka et al., 1993; Takayanagi et al., 2002). MCSF-1 enhances osteoclast proliferation during RANKL-induced osteoclasts generation (Biskobing et al., 1995). The osteoclast-activating transcription factors c-Fos and NFATc1 are also expressed by RANKL, which leads to expression of osteoclast associated receptor (OSCAR), tartrate-resistant acid phosphatase (TRAP), and other proteins (Teitelbaum and Ross, 2003).

Osteoporosis weakens bones, increasing risk of fracture. Osteoporosis is classified into two types, depending on the cause: estrogen-dependent osteoporosis occurs mainly in postmenopausal woman, and age-dependent osteoporosis occurs in people older than 70 years (Lim et al., 2009). The effects of herbal products and traditional foods on enhanced mineral 
accumulation in bones and maximization of bone mass in premenopausal and postmenopausal women have been studied. Traditional herbal medicines and natural materials have been investigated as possible cures for osteoporosis because of their inhibition of osteoclast differentiation with few side effects (Putnam et al., 2007). The effects of individual components of ginseng extract on osteoporosis have been examined. Ginsenosides Rb1, Rb2, and Rg3 affect osteoclasts that influence bone homeostasis (Cheng et al., 2012; Huang et al., 2014; Siddiqi et al., 2015).

Here, we used a zebrafish scale system to evaluate if ginseng and its bioactive components contributed to bone homeostasis. A major ginsenoside of aqueous ginseng extract, ginsenoside Re, exhibited novel effects in inhibiting osteoclast differentiation.

\section{MATERIALS AND METHODS}

\section{Reagents}

Culture media, fetal bovine serum, and antibiotics were from Invitrogen (USA), while standard ginsenosides (including Re), anti- $\beta$-actin antibody, and TRAP-staining solution were from Sigma (USA). Recombinant macrophage colony stimulating factor (MCSF) was from PeproTech EC (UK). Anti-c-Fos and anti-NFATc1 were from Santa Cruz Biotechnology, Inc. (USA). Recombinant GST-RANKL was purified from an Escherichia coli expression system.

\section{Preparation of aqueous ginseng extract}

Aqueous ginseng extract was from the National Agricultural Cooperative Federation in Chungbuk, Republic of Korea. To mimic intake conditions for traditional oriental herbs, we used a previously described water extraction process (Lee et al., 2015).

\section{Differentiation of mouse bone marrow-derived} macrophages and osteoclasts

Bone marrow-derived macrophages (BMMs) were obtained from femurs and tibias of 6-week-old ICR mice as described previously (He et al., 2012). To generate osteoclasts, BMMs (5 $\times 10^{4}$ cells $/$ well) were incubated with MCSF $(30 \mathrm{ng} / \mathrm{ml})$ and RANKL $(25 \mathrm{ng} / \mathrm{ml})$ in 48-well $(1 \mathrm{ml} /$ well) tissue culture dishes with or without ginsenoside $\mathrm{Re}$ or ginseng extract. Cells were fixed with $10 \%$ formalin for 10 min and washed 4 times with PBS plus $0.1 \%$ Triton X-100 (PBST). Cells were incubated for a few hours in TRAP staining solution (Sigma-Aldrich).

\section{Western blots}

Cells were lysed in lysis buffer [PBS plus $0.5 \%$ NP-40, 0.5 $\mathrm{mM} \mathrm{Na}_{3} \mathrm{VO}_{4}, 20 \mathrm{mM}$-Nitrophenyl Phosphate (PNPP)]. Equal amounts of lysate $(30 \mu \mathrm{g})$ were separated by $8 \%$ to $10 \%$ sodium dodecyl sulfate-polyacrylamide gel electrophoresis (SDS-PAGE) and transferred to PVDF membranes. The resulting membranes were immunoblotted with the indicated antibodies.

\section{Experimental animals and treatments}

All experimental procedures complied with the act on life ethics and safety of the Ministry of Health and Welfare of South Korea. Wild-type adult zebrafish were maintained at $27.0^{\circ} \mathrm{C} \pm 1.0^{\circ} \mathrm{C}$ under light conditions of $14 \mathrm{~h}$ light and $10 \mathrm{~h}$ dark. Before starting the experiments, the weight of zebrafish more than $350 \mathrm{mg}$ (similar in length) were maintained for 14 days in the same cage. To induce bone loss from fish scales, we reduced the feeding amount of food by $20 \%$. Ten zebrafish per each group were separated individually in standard cages. For 7 days, the fish were raised without feeding in the presence or absence of ginsenoside $\mathrm{Re}$ and other 28 days, the fish were fed $2 \mathrm{ml}$ $(20 \%)$ of artemia one time a day in water, after $1 \mathrm{hr}$ the water was replaced to half volume of fresh water with or without of ginsenoside Re every day to maintain optimal water condition. 35 days later, the fish were anaesthetized with $0.01 \%$ tricaine methanesulfonate (Sigma) before scale collection; the scales were carefully removed from either side of the body under a stereomicroscope (KL300, Leica, Germany) using forceps.

\section{Cell viability assays}

Cell cytotoxicity assays were performed as described previously (He et al., 2012). Cells were cultured with or without aqueous ginseng extract $(0-100 \mu \mathrm{g} / \mathrm{ml})$ or ginsenoside $\operatorname{Re}(0-10 \mu \mathrm{M})$ for $48 \mathrm{~h}$. After $1 \mathrm{~h}$ of Cell Counting Kit -8 (CCK-8) treatment (10 $\mu \mathrm{l})$, plates were analyzed at $450 \mathrm{~nm}(650 \mathrm{~nm}$ reference) using a 96-well plate recorder (Molecular Devices, USA).

\section{TRAP staining and activity assays}

Multinucleated osteoclasts were treated with $10 \%$ formalin for $10 \mathrm{~min}$ and ethanol/acetone (50\%/50\%) for $1 \mathrm{~min}$ for fixation and then stained with TRAP staining solution (Sigma-Aldrich). TRAP-positive multinucleated cells were photographed under a microscope (Olympus Optical Co. Ltd., Japan). To measure TRAP activity, cells were fixed with $10 \%$ formalin for $10 \mathrm{~min}$ and $95 \%$ ethanol for $1 \mathrm{~min}$ and then incubated with $100 \mu \mathrm{l}$ reaction buffer ( $50 \mathrm{mM}$ citric acid, $\mathrm{pH} 4.6,10 \mathrm{mM}$ sodium tartrate, 5 $\mathrm{mM}$ PNPP). After $1 \mathrm{~h}, 30 \mu \mathrm{l}$ was transferred to a new plate containing an equal volume of stopping buffer $(0.1 \mathrm{~N} \mathrm{NaOH})$. Absorbance was measured at $410 \mathrm{~nm}$. TRAP activity was presented as a percentage of the control.

\section{RNA preparation and RNA quantitation by RT-PCR}

RNA preparation and cDNA synthesis were performed as described previously (He et al., 2012). Real-time polymerase chain reaction (PCR) used a CFX96 ${ }^{\mathrm{TM}}$ Real-time system with SYBR FAST KAPA iCycler qPCR kit under the following conditions: 40 cycles of denaturation at $95^{\circ} \mathrm{C}(15 \mathrm{~s})$ and amplification at $60^{\circ} \mathrm{C}(1 \mathrm{~min})$. All reactions were run in triplicate, and the data were normalized to that of $\beta$-actin. Relative differences in PCR results were evaluated using the comparative cycle threshold method. Primer sets were: mouse NFATc1, 5'-CCGTTGCTTCCAGAAAATAACA-3' (forward), 5'-TGTGGGATGTGAACTCGGAA-3' (reverse); mouse $\beta$-actin, 5'-TCTGCTGGAAGGTGGACAGT-3' (forward), 5'-CCTCTATGCCAACACAGTC-3' (reverse); mouse TRAP 5'-CTGGAGTGCACGATG-CCAGCGACA-3' (forward), 5'-TCCGTGCTCGGCGATGGACCAGA-3' (reverse); zebrafish cathepsin $\mathrm{K}, 5^{\prime}$-CTATTAAAGAGATTCCTCAGGGTAAGCA-3' (forward), 5'- ACACGGGTCCCACATTGG-3' (reverse); zebrafish TRAP, 5'- CGTCCACTGACCACAGGAAGA-3' (forward), 5'-AAGGA-TCCTGACGTCTGATTGA3' (reverse); zebrafish matrix metalloproteinase (MMP)-9, 5'AAATCTGTGTTCGTGACGTT-TCCT-3' (forward), 5'-GCCGTAACGCTT-CAGATACTCAT-3' (reverse); zebrafish MMP2, 5'TTGCTTCCCTGCAAACTTTTG-3' (forward), 5'-GAGCCACTTCTTTGTCTGTGTGA-3' (reverse); zebrafish alkaline phosphatase, 5'-CAGTGGGAATCGTCACAACAA-3' (forward), 5'CCACACAGTGGGCATAAGCA-3' (reverse); zebrafish osterix, 5'-AAGAAACCTGTCCACAGCTG-3' (forward), 5'- GAGGCTTTACCGTACACCTT-3' (reverse); and zebrafish $\beta$-actin, $5^{\prime}$ CAACAGGGAAAAGATG-ACACAGAT-3' (forward), 5'-CAGCCTGGATGGCAACGT-3' (reverse). 
A
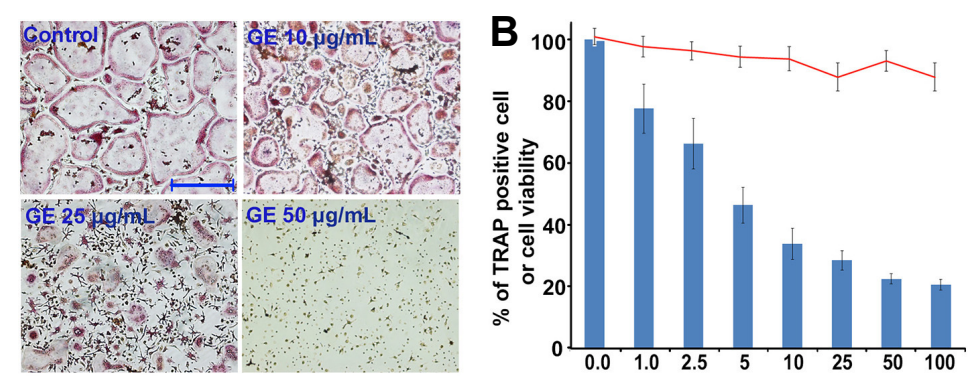

C

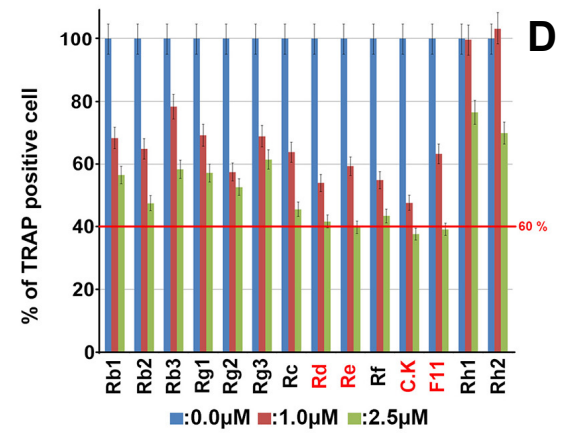

\begin{tabular}{c|cc}
\hline Ginsenoside & $\mathrm{EC}_{50}(\mu \mathrm{M})$ & $\mathrm{IC}_{50}(\mu \mathrm{M})$ \\
\hline $\mathrm{Rb} 1$ & 1.6 & 2.9 \\
$\mathrm{Rb} 2$ & 3.5 & $\mathrm{ND}$ \\
$\mathrm{Rb3}$ & 2.7 & $\mathrm{ND}$ \\
$\mathrm{Rg} 1$ & 3.7 & $\mathrm{ND}$ \\
$\mathrm{Rg} 2$ & $>5.0$ & $\mathrm{ND}$ \\
$\mathrm{Rg} 3$ & 4.7 & $\mathrm{ND}$ \\
$\mathrm{Rc}$ & 3.3 & $\mathrm{ND}$ \\
$\mathrm{Rd}$ & 2.0 & 2.8 \\
$\mathrm{Re}$ & 2.2 & $\mathrm{ND}$ \\
$\mathrm{Rf}$ & $>5.0$ & $\mathrm{ND}$ \\
Compund K & 1.4 & $\mathrm{ND}$ \\
Pseudo-F11 & $>5.0$ & $\mathrm{ND}$ \\
$\mathrm{Rh1}$ & $>5.0$ & $\mathrm{ND}$ \\
$\mathrm{Rh} 2$ & $>5.0$ & $\mathrm{ND}$
\end{tabular}

Park et al., Figure 1 a-d
Fig. 1. Aqueous ginseng extract inhibition of osteoclast differentiation, and effects of 14 ginsenosides on osteoclast differentiation. (A) Mouse BMMs were harvested and exposed for 3 days to RANKL (25 $\mathrm{ng} / \mathrm{ml})$ and M-CSF $(10 \mathrm{ng} / \mathrm{ml})$ with or without ginseng active compounds. TRAP staining was performed, and (B) TRAP activity was measured at $\lambda=405 \mathrm{~nm}$. Data are expressed as the percentage of TRAP activity in ginseng extract treated cells compared to untreated cells (mean \pm SEM, $n=3$ ) in blue, with cell viability at the same ginseng concentration tested by MTT assay in red. (C) RANKLinduced BMMs were treated with 14 ginsenosides, and osteoclast differentiation was evaluated by TRAP activity measured at $\lambda=405 \mathrm{~nm}$. Data are expressed as a percentage of TRAP activity of untreated cells (mean \pm SEM, $n=3$ ). The red line indicates $60 \%$ inhibition at $2.5 \mu \mathrm{M}$. More effective ginsenosides are indicated in red. (d) Summary of osteoclast-differentiation inhibition efficiency of 14 ginsenosides and cell viability by MTT assays. $\mathrm{EC}_{50}$, half-maximal effective concentration. $\mathrm{IC}_{50}$, half-maximal growth inhibition concentration. Scale bar, $100 \mu \mathrm{m}$.
Microcomputed tomography scanning of zebrafish bone To measure zebrafish vertebrae density, fish were fixed in a stretched position on a sample holder and scanned with a microcomputed tomography ( $\mu-\mathrm{CT}$ ) system (Siemens, USA). Experiments were performed on an animal Inveon system in the Ochang Center at the Korea Basic Science Institute. The $\mu-$ CT scanner was set to $80 \mathrm{kVp}$ for the $\mathrm{X}$-ray tube, $500 \mu \mathrm{A}$ for the $\mathrm{X}$-ray source, and 800-ms exposure time. The detector and Xray source were rotated $360^{\circ}$ in 360 steps. The number of calibration exposures was 30 . The system magnification was set to $102.55 \mathrm{~mm}$ axial field view and $30.74 \mathrm{~mm}$ transaxial field view. Data were analyzed using Inveon Research Workplace software (Siemens).

\section{Measurement of calcium/phosphorus ratio in scales}

To measure the calcium and phosphorus concentration in bones (vertebrae) and scales, we prepared the scales and vertebrae from each group, the scales were collected by forceps from each side of fish. The remaining body was taken off head and then treated $0.1 \%$ trypsin for $10 \mathrm{~min}$ at 37 to collect the vertebrae. The scales and vertebrae were washed 2 times with distilled water. Both samples were measured the weight after incubating in dry oven for overnight, and then the samples were measured by atomic absorption spectrometry. Calcium/phosphorus (Ca:P) ratios in samples were measured by inductively coupled plasma mass spectrometry (VG PlasmaQuad, Fisons Instruments, UK). All values were adjusted for minor deviations from standard calcium solutions with accepted natural ratios (the ratio of $\mathrm{Ca}: P$ is about 2:1). All samples were measured in duplicate (van den Heuvel et al., 2000).

\section{Statistical analysis}

Values are presented as mean \pm standard deviation (SD) of three or more experiments. Data were analyzed using Student's $t$-test or one-way analysis of variance for comparisons between two mean values. A value of $P<0.05$ was considered significant.

\section{RESULTS}

Inhibition effect of aqueous ginseng extract on RANKLinduced osteoclast differentiation in mouse BMMs

To test the effect of aqueous ginseng extract on osteoclast differentiation, cells were isolated from mice and incubated with the osteoclast-generating factors MCSF-1 and RANKL, with or without aqueous ginseng extract. After TRAP staining, TRAPpositive multinucleated cells were counted. Aqueous ginseng extracts dose-dependently diminished osteoclast differentiation (Figs. 1A and 1B) without toxicity up to $100 \mu \mathrm{g} / \mathrm{ml}$ (Fig. 1B). The same procedure was used to assay 14 types of ginsenosides at various concentrations. At $2.5 \mu \mathrm{M}$, ginsenosides $\mathrm{Rd}, \mathrm{Re}$, and compound K; and Fraction 11 inhibited osteoclast differentiation by $\sim 60 \%$ (Fig. 1C). The ginsenoside Rd showed toxicity (Fig. 1D). Among individual ginsenosides, ginsenoside Re exhibited the most potent inhibition of osteoclast differentiation.

\section{Effect of aqueous ginseng extract on zebrafish model}

We investigated the effects of 30 days of limited feeding on our zebrafish model. Zebrafish vertebrae were examined ex vivo by $\mu-\mathrm{CT}$. The $\mu$-CT images showed that our experimental conditions did not significantly affect the vertebrae (Fig. 2A). The $\mathrm{Ca}: \mathrm{P}$ ratio was in the normal range $(2: 1)$ in control vertebrae samples compared with the samples treated with aqueous ginseng extract. In samples of scales, which are part of the fish skeleton, the Ca:P ratio was significantly decreased to 1.78:1. However, the Ca:P ratio was restored to $2.06: 1$ in samples treated with aqueous ginseng extract (Fig. 2b). We also found that the mRNA expression level of the osteoclast marker genes including TRAP, MMP9, and MMP2 was diminished; in particular, the TRAP expression was much lower in aqueous ginseng extract-treated samples compared to the controls (Fig. 2C) 

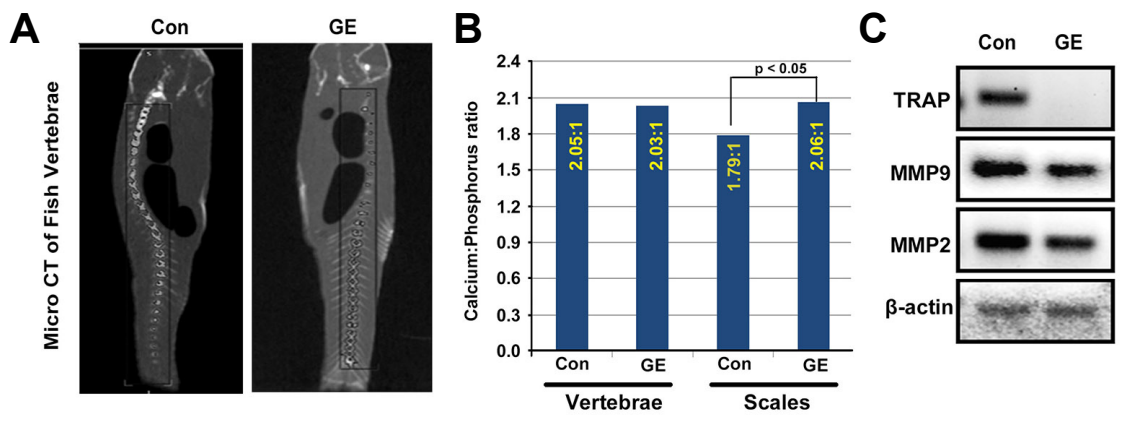

D
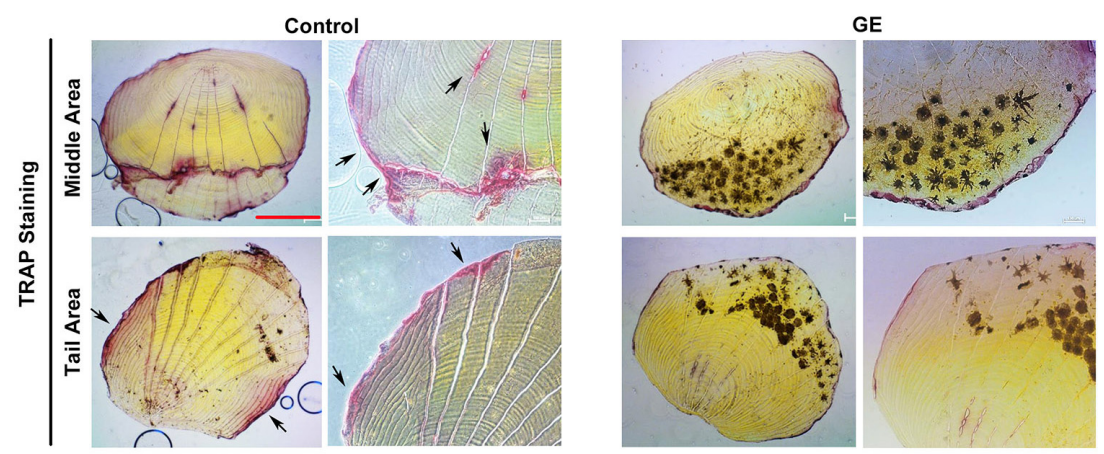

Park, et al., Figure 2 a-d
Fig. 2. Effect of aqueous ginseng extract on osteoporosis in the zebrafish scale model. (A) After treatment with aqueous ginseng extract (GE, $10 \mu \mathrm{g} / \mathrm{ml}$ ) for 35 days, zebrafish were scanned by $\mu-C T$ to visualize vertebrae density. (B) Calcium/phosphorous ratios were measured in vertebrae and scales from zebrafish samples by inductively coupled plasma mass spectrometry. (C) RT-PCR was performed to determine the mRNA expression levels of osteoclast marker genes TRAP, MMP9, and MMP2 of and $\beta$-actin. (D) Zebrafish scales were stained with TRAP. Scale bar, $100 \mu \mathrm{m}$.

\section{A}

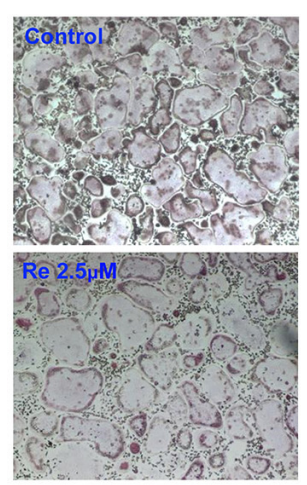

D

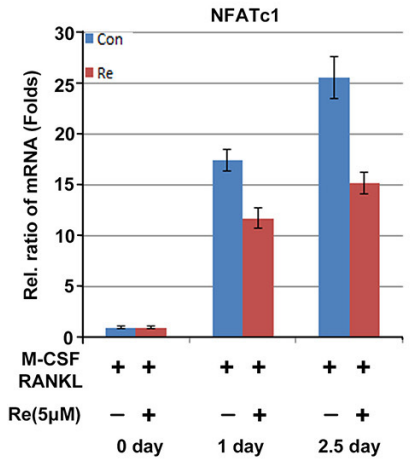

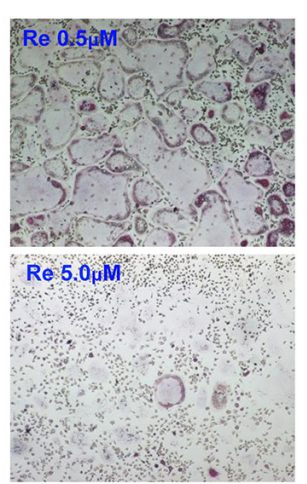

E

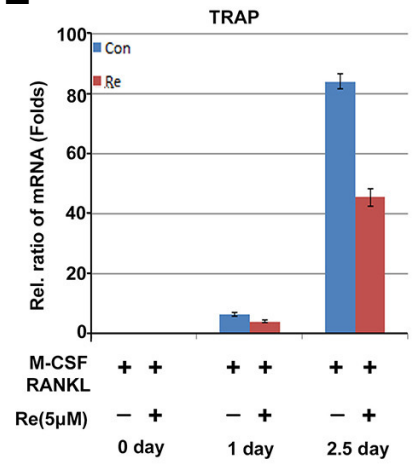

C

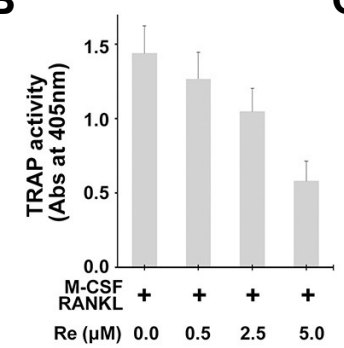

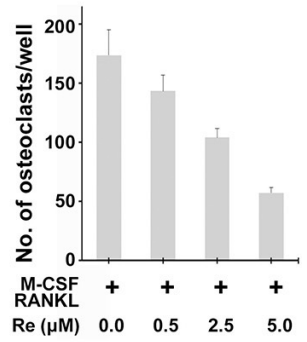

$\mathbf{F}$

Park et al., Figure 3 a-f

Fig. 3. Effect of ginsenoside Re on osteoclast differentiation. (A) Total osteoclast formation by BMMs treated with the indicated concentrations of ginsenoside Re. TRAP staining was performed after 3 days to show TRAP + multinucleated cells, which were rare in the concentration of 5 $\mu \mathrm{M}$ groups. 40x. (B) TRAP activity was measured at $\lambda=405 \mathrm{~nm}$. Data are expressed as a percentage of the TRAP activity of untreated cells (means $\pm S E M, n=3$ ). (C) TRAP-positive cells were counted under the same conditions. (D, E) qPCR was performed to confirm the mRNA expression levels of NFATC1 and TRAP genes. (F) Cell viability at the indicated concentration of ginsenoside Re was tested by MTT assay. 
To confirm these findings, we visualized zebrafish scales with TRAP staining to examine the inhibition of osteoclast differentiation. Based on the results, we concluded that treatment with aqueous ginseng extract decreased osteoclast differentiation activity. Compared with controls, TRAP signal intensity was lower in aqueous ginseng extract-treated samples (Fig. 2D).

\section{Effect of ginsenoside Re on osteoclast differentiation}

Ginsenoside $R e$ is a major compound in aqueous ginseng extract (Lee et al., 2015). To elucidate the effects of ginsenoside $\mathrm{Re}$, it was applied to BMMs with RANKL $(25 \mathrm{ng} / \mathrm{ml})$ and MCSF $(30 \mathrm{ng} / \mathrm{ml})$ at indicated concentrations and times. Based on TRAP activity assays and positive-osteoclasts cell counting, ginsenoside Re dose- dependently inhibited osteoclast differentiation (Figs. 3A-3C). Additionally, mRNA levels of the osteoclast differentiation marker genes NFATc1 and TRAP decreased up to $50 \%$ in 2.5 days (Figs. 3D and 3E). These results were from tests using $5 \mu \mathrm{M}$ ginsenoside Re, which did not affect cell viability (Fig. 3f).

\section{Blocking effect of ginsenoside Re on RANKL-induced osteoclast differentiation signaling pathway}

To explore the mechanisms by which ginsenoside $\mathrm{Re}$ inhibits osteoclast differentiation, we tested whether ginsenoside Re reduced protein levels of the transcription factors NFATc1 and c-Fos in BMMs stimulated with RANKL. The levels of the protein degradation control protein CYLD did not change with ginsenoside Re treatment (Fig. 4A). RANKL-induced differentiation of osteoclasts involves amplification of cell signaling mediated through mitogen-activated protein kinases and activation of downstream transcription factors such as NF- $\mathrm{KB}$ and NFATc1 (Wei et al., 2002).

Next, we investigated the effects of ginsenoside Re on intracellular signaling pathways in RANKL-induced BMMs. RANKL treatment of BMMs for 5 min dramatically increased phosphorylation of extracellular signal-regulated kinase (ERK); this was inhibited by treatment with ginsenoside Re (Figs. 4B and $4 C$ ). RANKL-induced p38 and JNK activation was not influenced by ginsenoside $R e$ in the same conditions (Figs. 4B, 4D, and 4E). And we further examined the effect of ginsenoside Re on NF$\kappa \mathrm{B}$ signaling pathway, which is another major signaling pathway in RANKL-induced osteoclast differentiation. The phosphorylation-dependent degradation of $\mathrm{I}_{\kappa} \mathrm{Ba}$, a negative feedback regulator of NF- $\mathrm{KB}$, is a key event in RANKL-induced osteoclast differentiation and it is the evidence of NF- $\mathrm{KB}$ activation (Boyce et al., 2015). Ginsenoside Re-treated BMMs exhibited significantly decreased the amounts of total $I_{\kappa} B \alpha$ level same as DMSO treated samples even though RANKL-induced phospho-IkBa level was remained slightly in ginsenoside $\mathrm{Re}$ treated samples (Figs. 4F and 4G). Thus, the inhibitory effects of ginsenoside $R e$ on osteoclastogenesis were shown to be mediated by blocking via ERK signaling pathway, not NF-kB pathway.

\section{Effect of ginsenoside Re on osteoclast differentiation in zebrafish scales}

We investigated the effects of ginsenoside Re as a major component of aqueous ginseng extract (Fig. 2). Based on toxicity tests using 3-(4, 5-dimethylthiazol-2-yl)-2,5-diphenyltetrazolium bromide (MTT) assays (Fig. 3F), we selected a concentration of $5 \mu \mathrm{M}$ as the optimal concentration for the zebrafish scale model. To confirm that $5 \mu \mathrm{M}$ ginsenoside Re was appropriate for treatment in our model, we treated fish embryos for 3 days with 0 to $10 \mu \mathrm{M}$ ginsenoside Re. In toxicity tests with zebrafish em-
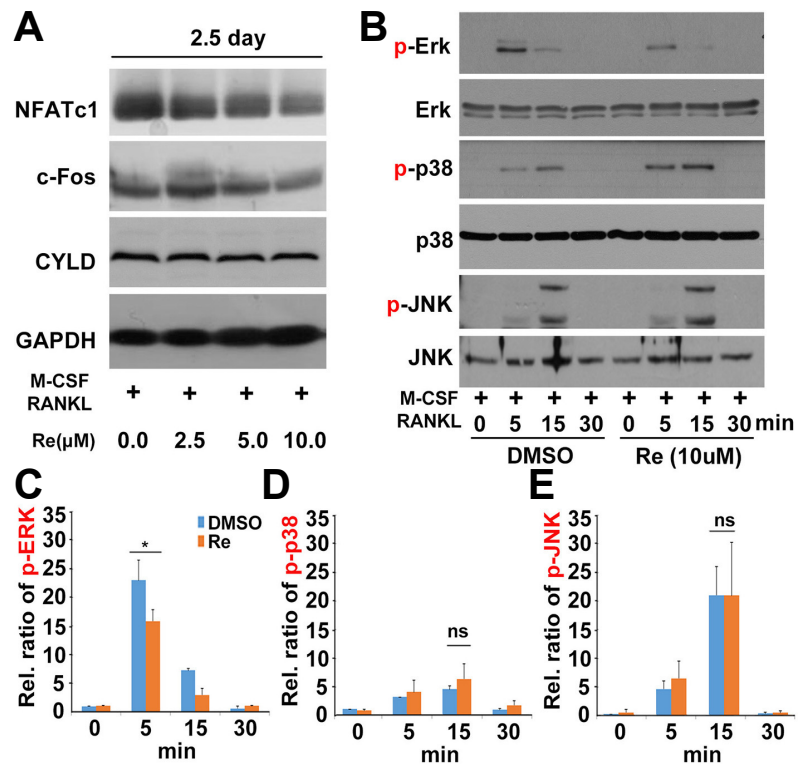

E

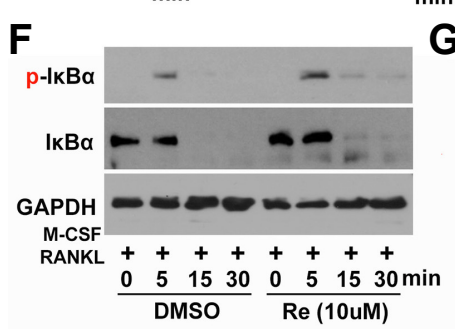

G

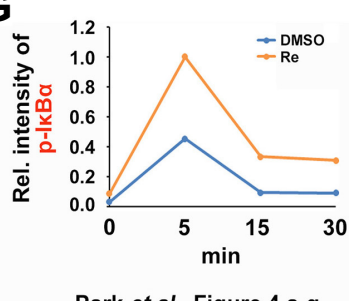

Park et al., Figure $4 \mathrm{a}-\mathrm{g}$

Fig. 4. Inhibition of ERK and NF- $\kappa B$ pathways by ginsenoside $R e$ in BMMs. (A) BMMs were treated with ginsenoside $\operatorname{Re}(0-10 \mu \mathrm{M})$ with M-CSF $(30 \mathrm{ng} / \mathrm{ml})$ and RANKL $(25 \mathrm{ng} / \mathrm{ml})$ for 2.5 days. Whole cell lysates were extracted for Western blotting using antibodies against NFATc1, c-Fos, and CYLD. (B, F) BMMs were treated with ginsenoside Re $(10 \mu \mathrm{M})$ or control (DMSO) for the indicated times. Whole cell lysates were used for Western blotting with antibodies against p-ERK, ERK, p-p38, p38, p-JNK, JNK, p-IкBa, and IкBa. GAPDH was the reference protein. (C, D, E, and G) The intensities of $p$-ERK, p-p38, p-JNK and $p$ - IкBa were measured by densitometer. ${ }^{*} P<0.05$ by one-way ANOVA.

bryos, the highest concentration $(10 \mu \mathrm{M})$ showed little toxicity such as embryonic death or abnormal morphology (data not shown). We used TRAP assays to evaluate the efficiency of inhibition of osteoclast differentiation on fish scale samples from controls and fish treated with ginsenoside Re for 35 days. Control scales showed TRAP staining that was widely distributed in the central and lateral areas, but ginsenoside Re-treated scales showed a more narrow distribution of TRAP staining (Fig. 5A). TRAP intensity for ginsenoside Re-treated samples was only 0.37-fold compared to the controls (Fig. 5B). This result indicates that ginsenoside Re suppressed osteoclast generation by zebrafish scales. These observations were confirmed by the decreased expression of the osteoclast marker genes TRAP and cathepsin $K$; gene expression was decreased $\sim 0.7-0.8$ fold in ginsenoside Re-treated samples compared to the controls (Figs. 5C and 5D). Expression of the osteoblast marker genes ALP and osterix, however, was the same in the control and ginsenoside Re samples (Figs. 5E and 5F). 
A

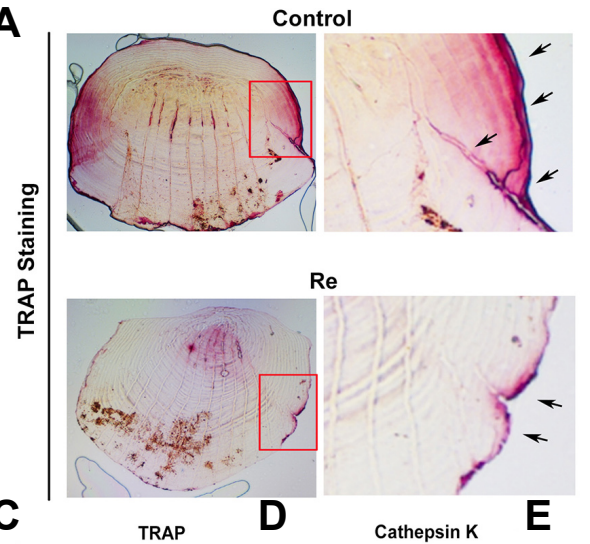

B
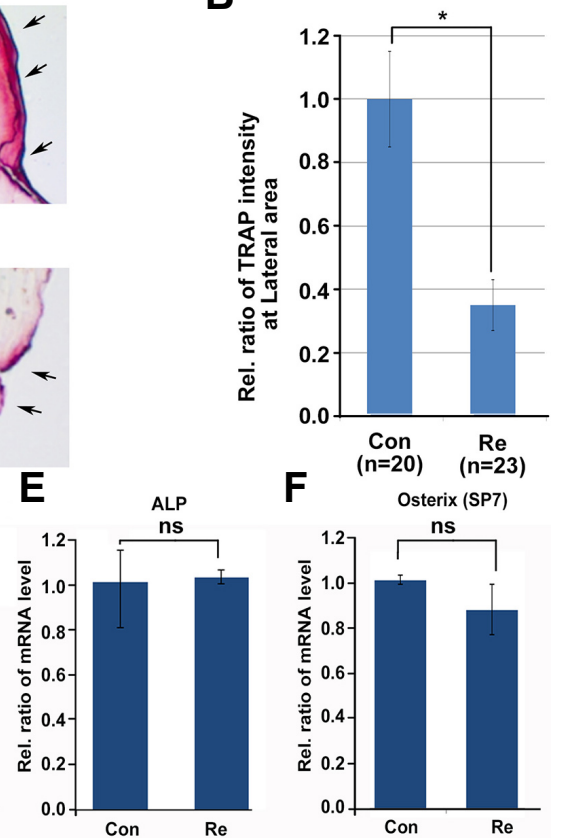

$\mathbf{F}$

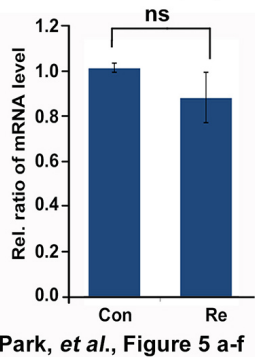

Fig. 5. Effect of ginsenoside Re on zebrafish scale osteoclasts. (A) Ginsenoside Re-treated zebrafish scales were stained with TRAP. Red box, magnified X200; arrow, TRAP-positive signal. (B) TRAP signal intensity was measured in lateral regions. Five randomly selected points were measured per treated scale. $(C, D)$ qPCR was performed to determine the mRNA levels of the osteoclast markers TRAP and cathepsin $K$ and $(\mathrm{E}, \mathrm{F})$ the osteoblasts markers $A L P$ and osterix. ${ }^{\star} P<0.05$ and ${ }^{\star \star} P<0.01$ by student's $t$-test.

\section{DISCUSSION}

Bone health depends on the balance between osteoblasts and osteoclasts. In this study, we found that aqueous ginseng extract negatively regulated osteoclastogenic activity, with both in vitro and in vivo evidence of the anti-osteoporotic effects of the extract. Aqueous ginseng extract effectively suppressed osteoclast differentiation in mouse BMMs and zebrafish scales. Low $\mathrm{Ca}: \mathrm{P}$ ratios affects serum parathyroid hormone (PTH) concentration and increases bone resorption (Kemi et al., 2010). Even through the major bone system, the vertebrae were not changed much, the Ca:P ratio of the scales treated with aqueous ginseng extract was significantly increased calcium uptake to naturally acceptable ratio $(2.06: 1)$ compared with the control (1.79:1), which suggested that ginseng extracts have an effects on inhibition of bone resorption by osteoclast differentiation (Fig. 2).

Aqueous ginseng extract inhibited osteoclast differentiation without toxicity toward BMMs. Ginsenosides are reported to possess effects such as anti-oxidant, anti-cancer, anti-diabetic, anti-adipocyte, anti-osteoporotic, and sexual enhancement activities (Shin et al., 2006). Our group previously reported that red ginseng combined with other herbal medicines regulates osteoporosis in ovariectomized rats (Kim et al., 2008).

In this study, we report the efficacy of a single ginseng treatment for preventing bone resorption through suppression of osteoclast differentiation. Among the various ginsenosides, major ginsenoside in aqueous ginseng extract, ginsenoside $R e$ treatment blocked osteoclast differentiation and RANKLinduced NFATc1 expression level as osteoclastogenesis key transcription factor without any cytotoxicity in mouse BMMs (Fig. 3). In addition, we observed that ginsenoside Re influenced osteoclast differentiation via inhibition of RANKL-induced signaling pathway. In particular, ginsenoside Re blocked signaling of ERK and, which is known mechanism of osteoclast differentiation (Fig. 4). And we applied effect of ginsenoside Re to in vivo system on osteoclast differentiation using zebrafish model.

Osteoclast differentiation was inhibited by ginsenoside $R e$ and osteoclast marker genes were significantly decreased in samples treated with ginsenoside Re in zebrafish scales. Ginsenoside Re treatment decreased mRNA expression level of TRAP and cathepsin $K$, although it did not markedly affect expression of osteoblast marker genes (Fig. 5). These findings demonstrated that the zebrafish scale model was an experimental approach for testing the effects of ginseng extract or ginsenoside $\mathrm{Re}$ on osteoclast differentiation. Therefore, we suggest that ginseng extract and the major ginsenoside $\mathrm{Re}$ appear to have beneficial effects on bone health.

\section{ACKNOWLEDGMENTS}

This study was financially supported by the Medicinal Crops Division, Ginseng and Medicinal Plants Research Institute, Rural Development Administration (PJ010221) and by the KRIBB Research Initiative Program funded by the Ministry of Science, ICT, and Future Planning (MSIP), Republic of Korea.

\section{REFERENCES}

Biskobing, D.M., Fan, X., and Rubin, J. (1995). Characterization of MCSF-induced proliferation and subsequent osteoclast formation in murine marrow culture. J. Bone Miner. Res. 10, 1025-1032.

Boyce, B.F., Xiu, Y., Li, J., Xing, L., and Yao, Z. (2015). NF-kappaBmediated regulation of osteoclastogenesis. Endocrinol. Metabol. 30, 35-44.

Boyle, W.J., Simonet, W.S., and Lacey, D.L. (2003). Osteoclast differentiation and activation. Nature 423, 337-342.

Cheng, B., Li, J., Du, J., Lv, X., Weng, L., and Ling, C. (2012). Ginsenoside Rb1 inhibits osteoclastogenesis by modulating NFkappaB and MAPKs pathways. Food Chem. Toxicol. 50, 16101615.

Goltzman, D. (2002). Discoveries, drugs and skeletal disorders. Nat. Rev. 1, 784-796.

He, L., Lee, J., Jang, J.H., Lee, S.H., Nan, M.H., Oh, B.C., Lee, 
S.G., Kim, H.H., Soung, N.K., Ahn, J.S., et al. (2012). Ginsenoside Rh2 inhibits osteoclastogenesis through downregulation of NF-kappaB, NFATc1 and c-Fos. Bone 50, 12071213.

Huang, Q., Gao, B., Jie, Q., Wei, B.Y., Fan, J., Zhang, H.Y., Zhang, J.K., Li, X.J., Shi, J., Luo, Z.J., et al. (2014). Ginsenoside-Rb2 displays anti-osteoporosis effects through reducing oxidative damage and bone-resorbing cytokines during osteogenesis. Bone 66, 306-314.

Kemi, V.E., Karkkainen, M.U., Rita, H.J., Laaksonen, M.M., Outila, T.A. and Lamberg-Allardt, C.J. (2010). Low calcium:phosphorus ratio in habitual diets affects serum parathyroid hormone concentration and calcium metabolism in healthy women with adequate calcium intake. Br. J. Nutr. 103, 561-568.

Kim, H.R., Cui, Y., Hong, S.J., Shin, S.J., Kim, D.S., Kim, N.M., So, S.H., Lee, S.K., Kim, E.C., Chae, S.W., et al. (2008). Effect of ginseng mixture on osteoporosis in ovariectomized rats. Immunopharmacol. Immunotoxicol. 30, 333-345.

Kong, Y.Y., Yoshida, H., Sarosi, I., Tan, H.L., Timms, E., Capparelli, C., Morony, S., Oliveira-dos-Santos, A.J., Van, G., Itie, A., et al. (1999). OPGL is a key regulator of osteoclastogenesis, lymphocyte development and lymph-node organogenesis. Nature 397, 315323.

Kropotov, A.V., Kolodnyak, O.L., and Koldaev, V.M. (2002). Effects of Siberian ginseng extract and ipriflavone on the development of glucocorticoid-induced osteoporosis. Bull. Exp. Biol. Med. 133, 252-254.

Lee, T.K., O'Brien, K.F., Wang, W., Johnke, R.M., Sheng, C., Benhabib, S.M., Wang, T., and Allison, R.R. (2010). Radioprotective effect of American ginseng on human lymphocytes at 90 minutes postirradiation: a study of 40 cases. J. Altern. Complement. Med. 16, 561-567.

Lee, H.Y, Park, S.H., Chae, S.W., Soung, N.K., Oh, M.j., Kim, J.S., Kim, Y.O., and Chae, H.J. (2015). Aqueous ginseng extract has a preventive role in RANKL-induced osteoclast differentiation and estrogen deficiency-induced osteoporosis. J. Funct. Foods 13, 192-203.

Lim, Y.U., Sun, D.H., and Kim, Y.S. (2009). Etiological cause of osteoporosis and prevention of fracture by osteoporosis. J. Korean Hip Society 21.

Polan, M.L., Hochberg, R.B., Trant, A.S., and Wuh, H.C. (2004). Estrogen bioassay of ginseng extract and ArginMax, a nutritional supplement for the enhancement of female sexual function. J.
Women's Health 13, 427-430.

Putnam, S.E., Scutt, A.M., Bicknell, K., Priestley, C.M., and Williamson, E.M. (2007). Natural products as alternative treatments for metabolic bone disorders and for maintenance of bone health. Phytotherapy Res. 21, 99-112.

Shin, Y.W., Bae, E.A., Kim, S.S., Lee, Y.C., Lee, B.Y., and Kim, D.H. (2006). The effects of ginsenoside Re and its metabolite, ginsenoside Rh1, on 12-O-tetradecanoylphorbol 13-acetate- and oxazolone-induced mouse dermatitis models. Planta Medica 72, 376-378.

Siddiqi, M.H., Siddiqi, M.Z., Ahn, S., Kang, S., Kim, Y.J., Sathishkumar, N., Yang, D.U., and Yang, D.C. (2013). Ginseng saponins and the treatment of osteoporosis: mini literature review. J. Ginseng Res. 37, 261-268.

Siddiqi, M.H., Siddiqi, M.Z., Kang, S., Noh, H.Y., Ahn, S., Simu, S.Y., Aziz, M.A., Sathishkumar, N., Jimenez Perez, Z.E., and Yang, D.C. (2015). Inhibition of osteoclast differentiation by ginsenoside Rg3 in RAW264.7 cells via RANKL, JNK and p38 MAPK pathways through a modulation of cathepsin $\mathrm{K}$ : an in silico and in vitro study. Phytother. Res. [Epub ahead of print].

Takayanagi, H., Kim, S., Koga, T., Nishina, H., Isshiki, M., Yoshida, H., Saiura, A., Isobe, M., Yokochi, T., Inoue, J., et al. (2002). Induction and activation of the transcription factor NFATc1 (NFAT2) integrate RANKL signaling in terminal differentiation of osteoclasts. Dev. Cell 3, 889-901.

Tanaka, S., Takahashi, N., Udagawa, N., Tamura, T., Akatsu, T., Stanley, E.R., Kurokawa, T., and Suda, T. (1993). Macrophage colony-stimulating factor is indispensable for both proliferation and differentiation of osteoclast progenitors. J. Clin. Invest. 91, 257-263.

Teitelbaum, S.L., and Ross, F.P. (2003). Genetic regulation of osteoclast development and function. Nat. Rev. Genet. 4, 638649.

van den Heuvel, E.G., Schoterman, M.H., and Muijs, T. (2000). Transgalactooligosaccharides stimulate calcium absorption in postmenopausal women. J. Nutr. 130, 2938-2942.

Wei, S., Wang, M.W., Teitelbaum, S.L., and Ross, F.P. (2002). Interleukin-4 reversibly inhibits osteoclastogenesis via inhibition of NF-kappa B and mitogen-activated protein kinase signaling. J. Biol. Chem. 277, 6622-6630.

World Health Organization (2004). WHO scientific group on the assessment of osteoporosis at primary health care level. 